\title{
Qualidade de vida de estudantes de medicina e a dificuldade de conciliação do internato com os estudos
}

\author{
Quality of life of medicine students and the difficulty of conciliation of \\ clerkship and studies
}

Carolina Meyer', Diego Grasel Barbosa1, Rubian Diego Andrade¹, Geraldo Jose Ferrari Junior¹, Manoel Gomes Filho Neto², Adriana Coutinho Guimarães ${ }^{1}$, Érico Pereira Gomes Felden ${ }^{1}$

'Programa de Pós-Graduação em Ciências do Movimento Humano, Universidade do Estado de Santa Catarina (UDESC) - Florianópolis (SC), Brasil Departamento de Medicina Social, Centro de Ciências da Saúde, Universidade Federal de Alagoas (UFAL) - Maceió (AL), Brasil.

DOI: https://dx.doi.org/10.7322/abcshs.v44i2.1169

\begin{abstract}
RESUMO
Introdução: Os anos finais do curso de graduação em Medicina estão cercados pelo aumento gradual de responsabilidades acumuladas durante o curso, que por muitas vezes, podem sobrecarregar os estudantes modificando a qualidade de vida dos mesmos. Objetivo: Analisar o perfil de qualidade de vida de formandos do curso de Medicina e a sua associação com a dificuldade em conciliar o internato e os estudos. Métodos: A amostra foi formada por 302 estudantes, de ambos os sexos, com média de idade de 25,4 anos, que frequentavam o internato obrigatório do curso de Medicina. Foram investigadas a qualidade de vida por meio do WHOQOL-bref, informações sociodemográficas e características do internato. Resultados: Os scores da qualidade de vida geral $(63,52)$ e domínios Psicológico $(73,17)$ e Físico $(73,19)$ foram os indicadores de qualidade de vida com percepção mais negativa por parte destes estudantes. Da amostra, 29,1\% relataram percepção neutra ou negativa destas questões. Os domínios Físico $(p=0,004)$, Psicológico $(p=0,008)$ e Meio ambiente $(p=0,026)$ apresentaram poder preditivo de $15,8 \%$ da qualidade de vida geral. Além disso, os estudantes que possuíam dificuldades em conciliar o internato com o estudo tiveram menor qualidade de vida geral $(p=0,026)$ e física $(p=0,010)$. Conclusão: Os piores indicadores de qualidade de vida foram os domínios Psicológico, Físico e a qualidade de vida geral. Uma parcela da amostra relatou percepção neutra ou negativa de sua qualidade de vida. Alunos com dificuldade em conciliar o internato e os estudos apresentaram percepção mais negativa no domínio Físico e na qualidade de vida geral.
\end{abstract}

Palavras-chave: Qualidade de vida; medicina; estudantes de medicina.

\begin{abstract}
Introduction: The final years of the undergraduate medical course are surrounded by the gradual increase of responsibilities accumulated during the course, which can often overwhelm students by modifying their quality of life. Objective: To analyze the quality of life profile of graduates of the medical course and their association with the difficulty in conciliate clerkship and studies. Methods: The sample consisted of 302 students, with a mean age of 25.4 years. Were investigated the quality of life through the WHOQOL-bref, sociodemographic information and characteristics of clerkship. Results: The general quality of life (63.52) and the domains Psychological (73.17) and Physical (73.19) were the indicators more negative perception by students. In the sample, $29.1 \%$ reported neutral or negative perception of quality of life. The physical $(p=0.004)$, psychological $(p=0.008)$ and environment $(p=0.026)$ were predictive power of $15.8 \%$ of the overall quality of life. In addition, it was found that the students who had difficulties in conciliate the clerkship and the study had a lower general quality of life $(p=0.026)$ and physical quality $(p=0.010)$. Conclusion: The worst indicators of quality of life were the Psychological, Physical and general quality of life domains. A portion of the sample reported a neutral or negative perception of their quality of life. Students with difficulty to conciliate clerkship and studies show more negative perception in the physical domain and general quality of life.
\end{abstract}

Keywords: Quality of life; medicine; students, medical.

Recebido em: 22/05/2018

Revisado em: 26/10/2018

Aprovado em: 08/01/2019

Autor para correspondência: Érico Pereira Gomes Felden - Centro de Ciências da Saúde e do Esporte - Universidade do Estado de Santa Catarina Rua Paschoal Simone, 358 - Coqueiros - CEP: 88080-350 - Florianópolis (SC), Brasil - E-mail: ericofelden@gmail.com

Conflito de interesses: nada a declarar 


\section{INTRODUÇÃO}

A Organização Mundial da Saúde (OMS) trata a qualidade de vida como um parâmetro subjetivo que incorpora aspectos da saúde física, do estado psicológico, das relações sociais, de crenças e sua relação com o meio ambiente ${ }^{1}$. Esta amplitude do conceito também é discutida por Pereira et al. ${ }^{2}$ ao atentarem para a necessidade de, ao se discutir a qualidade de vida das pessoas, ser considerado quem a pessoa é no campo individual, como ela se ajusta ao contexto ao qual pertence, bem como o que faz para alcançar suas expectativas de vida.

$\mathrm{O}$ estudo de Figueiredo et al. ${ }^{3}$ indica que os profissionais da saúde nem sempre aplicam as estratégias que utilizam para promover a qualidade de vida do paciente em benefício próprio. Assim, em estudantes universitários da área da saúde, a qualidade de vida vem sendo objeto de recentes investigações ${ }^{4-6}$. Na área da Medicina, em especial, estudos vêm reportando resultados interessantes sobre o tema, como de Scholz et al. ${ }^{7}$ o qual observou que, embora estes estudantes entrem na universidade com uma boa qualidade de vida e estado de saúde, estas condições se deterioram ao longo da graduação. No sexto e último ano, no qual o treinamento em serviço é intensificado, há aumento na tensão relacionada à formatura e à responsabilidade pelo exercício pleno da profissão, assim como com a avaliação para a residência médica ${ }^{8}$.

Com relação à avaliação da qualidade de vida de universitários de Medicina, Zhang et al. ${ }^{9}$ identificaram diferenças significativas nos escores dos domínios físico, de saúde e das relações sociais considerando o período do curso, os alunos do terceiro ano, tiveram as piores pontuações. Da mesma forma, Chazan et al. ${ }^{8}$ em estudo com 394 universitários de Medicina revelaram que estar no terceiro ou sexto ano da graduação apresentou associação negativa com todos os domínios da qualidade de vida, principalmente sobre os domínios psicológico e relações sociais. O estudo de Diderichsen et al. ${ }^{10}$, no qual foram investigadas as expectativas de vida futura dos estudantes de Medicina do primeiro e último ano, de ambos os sexos, foi identificado que as preocupações acerca da qualidade de vida e lazer foram intensificadas nos alunos do último ano.

Assim, será que o déficit nas relações sociais e percepção psicológica em universitários do último ano de graduação de Medicina se devem em partes, pelo aumento gradual de responsabilidades acumuladas durante o curso, que por muitas vezes, podem sobrecarrega-los modificando a qualidade de vida dos mesmos? Diante do exposto, este estudo teve como objetivo analisar o perfil de qualidade de vida de formandos em Medicina e a sua associação com a dificuldade em conciliar o internato e os estudos.

\section{MÉTODOS}

A população constituiu-se de 508 estudantes de Medicina matriculados no internato médico de nove universidades de Santa
Catarina. O tamanho da amostra foi definido utilizando a proposta de Barbetta ${ }^{11}$, considerando um erro amostral de cinco pontos percentuais. A partir deste cálculo foi identificado o mínimo de 224 estudantes (homens e mulheres) para compor uma amostra representativa. Para isto, a seleção da amostra foi por conveniência e representativa da população.

A pesquisa de corte transversal foi desenvolvida com estudantes do curso de Medicina da Universidade Federal de Santa Catarina (UFSC) e de oito universidades privadas: Universidade do Sul de Santa Catarina (Unisul) - Campus Pedra Branca Palhoça e Campus de Tubarão; Universidade do Oeste de Santa Catarina (Unoesc); Universidade do Vale do Itajaí (Univali); Universidade da Região de Joinville (Univille); Universidade do Planalto Catarinense (Uniplac); Universidade Comunitária da Região de Chapecó (Unochapecó); Universidade do Extremo Sul Catarinense (Unesc). Todas as universidades localizavam-se no estado de Santa Catarina. Apenas uma universidade no Estado que oferece o curso de Medicina não autorizou a coleta, impossibilitando sua participação na pesquisa.

Os estudantes matriculados no último ano do curso de Medicina assinaram o Termo de Consentimento Livre e Esclarecido e responderam a um questionário autoaplicável, constituído de instrumentos previamente validados, de forma presencial ou online, sob a orientação dos pesquisadores. O questionário continha os seguintes blocos: a) Informações sociodemográficas: sexo, idade e fase do curso; b) Caracterização do internato: período de trabalho, duração da jornada diária, número de pacientes atendidos diariamente, plantões e dificuldades entre internato e estudo (no que tange a fase do curso de Medicina); c) Qualidade de vida: WHOQOL-bref ${ }^{12}$, que abrange questões dentro dos domínios físico, psicológico, social e ambiental. Resulta em escores que variam de 0 a 100, sendo que, quanto mais próximo de 100, melhor a qualidade de vida. A versão em português do WHOQOL-bref foi desenvolvida no Centro WHOQOL para o Brasil, no Departamento de Psiquiatria e Medicina Legal da Universidade Federal do Rio Grande do Sul, em Porto Alegre-RS/Brasil ${ }^{13}$ e totaliza 24 questões. Além dos quatros domínios, o instrumento contém duas perguntas sobre a qualidade de vida geral, "Como você avaliaria sua qualidade de vida?" e "Quão satisfeito(a) você está com a sua saúde?". As 24 questões abordam facetas sobre, respectivamente, dor, energia, sono, mobilidade, atividades diárias, dependência de medicação, capacidade de trabalho, sentimentos positivos, concentração, auto-estima, imagem corporal, sentimentos negativos, espiritualidade, relações pessoais, suporte social, atividade sexual, segurança física, ambiente, recursos financeiros, cuidados de saúde, aquisição de informações, participação em lazer, ambiente físico e transporte.

Esta pesquisa teve aprovação do Comitê de Ética em Pesquisa em Seres Humanos (CEPSH) da Universidade do Estado de Santa Catarina (UDESC) - Brasil, com protocolo número 333-11. Todos 
os procedimentos éticos para pesquisas com seres humanos foram obedecidos de acordo com a Declaração de Helsinki de 1975, revisada em 2000 e a Resolução 466/2012 do Conselho Nacional de Saúde do Brasil.

\section{Análise estatística}

A análise estatística foi efetuada com o software SPSS versão 20.0, por meio da estatística descritiva (frequência, percentual, média, desvio padrão, mediana e intervalo interquartil) e inferencial por meio do teste da regressão linear múltipla no modo Stepwise para analisar a contribuição dos domínios na variação da qualidade de vida geral. A distribuição dos resíduos do modelo múltiplo foi analisada por meio do teste Kolmogorov-Smirnov. Para verificar a diferença entre os grupos que possuem e não possuem dificuldades em conciliar o internato utilizou-se o teste de Kruskal-Wallis. Adotou-se um nível de probabilidade de significância de $5 \%$.

\section{RESULTADOS}

Foram analisados 302 formados de ambos os sexos do curso de Medicina de universidades do estado de Santa Catarina. A média de idade foi de $25,4( \pm 2,6)$ anos, com 134 sujeitos da $11^{\text {a }}$ fase e $168 \mathrm{da} 12^{\text {a }}$ que frequentavam a prática do internato obrigatório do último ano do curso de Medicina. A maior parte dos estudantes apresentou dificuldade em conciliar estudos e o internato $(65,2 \%)$, quase metade dos estudantes realizava o internato em todos os períodos $(48,8 \%)$ e possuía em média 9,29 2,47 horas em jornada diária do internato (Tabela 1).

As pontuações dos domínios do WHOQOL-bref, assim como as suas médias foram apresentadas na Figura 1. A média da avaliação da qualidade de vida geral foi de 63,52 pontos e as médias dos escores dos domínios foram: domínio psicológico 73,17 pontos; domínio meio ambiente 76,06 pontos; domínio físico com 73,19 pontos e com maior pontuação o domínio relações sociais com 78,29 pontos. De tal modo, que a qualidade de vida geral e os domínios psicológico e físico apresentaram as menores pontuações, indicando percepções mais negativas nestas questões no que tange a qualidade de vida (Figura 1).

Nas questões um e dois do WHOQOL-bref, investiga-se a percepção geral de saúde e qualidade de vida das pessoas. Com isso, as frequências de respostas às diferentes alternativas destas questões são apresentadas na Tabela 2. Foi possível verificar nesta análise que, embora a maior parte da amostra considerava sua qualidade de vida e saúde positivas (boa/muito boa; satisfeito/muito satisfeito), aproximadamente $1 / 3$ da amostra relatou percepção neutra ou negativa destas questões (Tabela 2).

A partir desta análise, fez-se necessário verificar se os domínios com menores e maiores pontuações são aqueles que melhor explicam a variação da qualidade de vida geral da amostra. A Tabela 3 expos esta análise a qual identificou que o domínio Relações sociais não apresentou contribuição significativa para a qualidade de vida geral. Em contrapartida, todos os demais foram relevantes $(\mathrm{p}<0,001)$ com uma capacidade preditiva da qualidade de vida geral de $15,8 \%$. Desta forma, os indicadores de qualidade de vida com menores scores foram os que melhor definiram a percepção de qualidade de vida dos formandos em Medicina (Tabela 3).

Para considerar as questões gerais e os domínios do WHOQOL-bref, foram verificados as médias em cada uma das questões do WHOQOL-bref com o intuito de compreender com mais profundidade a qualidade de vida dos estudantes de Medicina. As questões do WHOQOL-bref que apresentaram percepção mais negativa (pontuações mais baixas) referem-se

Tabela 1: Descrição da amostra dos estudantes de Medicina.

\begin{tabular}{|l|c|}
\hline Variáveis & Grupo geral \\
\hline Idade, anos & $25,4 \pm 2,5$ \\
\hline Sexo, \% & \\
\hline Homens & 44,4 \\
\hline Mulheres & 55,6 \\
\hline Fase do curso atual, \% & 39,1 \\
\hline $11^{\circ}$ fase & 60,9 \\
\hline $12^{\circ}$ fase & \\
\hline Faz plantão, \% & 66,3 \\
\hline Sim & 33,7 \\
\hline Não & $9,29 \pm 2,47$ \\
\hline Duração da jornada diária do internato, horas & $11,4 \pm 6,5$ \\
\hline Média de pacientes atendidos, por dia & \\
\hline Período de trabalho, \% & 4,0 \\
\hline Matutino & 5,4 \\
\hline Vespertino & 2,0 \\
\hline Noturno & 39,8 \\
\hline Matutino e vespertino & 48,8 \\
\hline Todos os períodos & 65,2 \\
\hline Dificuldade em conciliar internato e estudo & 34,8 \\
\hline Sim & 302 \\
\hline Não & \\
\hline Total, n & \\
\hline
\end{tabular}

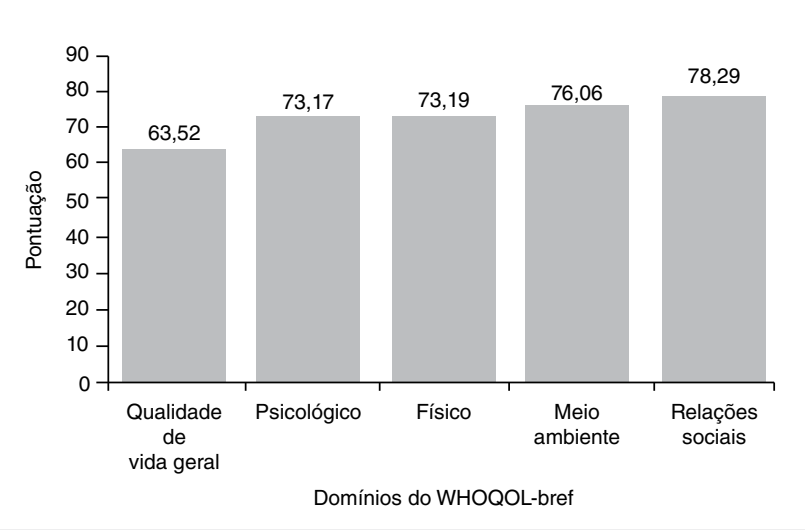

Figura 1: Pontuação média da avaliação da qualidade de vida nos domínios do WHOQOL-bref. 
a situações relacionadas ao domínio Psicológico (como ansiedade e depressão). As demais questões com piores pontuações foram nos domínios Físico e Meio ambiente, que se referem a problemas com sono, segurança, qualidade do ambiente físico e necessidade de tratamento médico. Por outro lado, as questões com maiores pontuações estão ligadas a possibilidades de obter informações e acesso aos serviços de saúde (alocadas no domínio Físico); sentido para a vida e satisfação com a autoimagem (alocadas no domínio Psicológico) e capacidade para o trabalho (alocadas no domínio Físico). As questões do domínio relações sociais não foram alocadas nos grupos de melhores e piores pontuações, pois não possuía capacidade explicativa da qualidade de vida geral (Tabela 4).

Além disso, foi observado que os estudantes que afirmaram possuir dificuldades em conciliar o internato com o estudo tiveram menor qualidade de vida, especialmente, no domínio físico $(\mathrm{p}=0,026)$ e na qualidade de vida geral $(\mathrm{p}=0,010)$ (Tabela 4$)$.

\section{DISCUSSÃO}

A relação positiva entre qualidade de vida e formação médica é fundamental para o exercício pleno da futura profissão. Na medida em que se trata de uma profissão relevante para a saúde da população, é esperado que estes profissionais apresentem boa qualidade de vida considerando que isto pode influenciar diretamente também na qualidade do tratamento e atendimento ao paciente. Apesar da educação médica ser longa na duração e consistir em uma grande pressão acadêmica ${ }^{14}$, supõe-se que os universitários com qualidade de vida mais positiva no período de internato, transfiram esta positividade para o dia-a-dia da profissão.

A média da qualidade de vida geral do presente estudo foi de 63,5 pontos. Em estudo de Nayak et al. ${ }^{14}$, com 250 estudantes de Medicina indianos do segundo e último ano, foi identificada média geral da qualidade de vida de 75,1 pontos, resultado semelhante ao observado nos estudantes de Santa Catarina (média de 63,3 pontos). Confirmando este perfil de qualidade de vida, Bampii et al. ${ }^{15}$ investigaram a qualidade de vida de 84 estudantes de Medicina de Brasília - DF de todos os semestres, identificaram que o domínio mais bem avaliado foi o das relações sociais e o domínio com pior escore de avaliação foi o psicológico, corroborando com os achados do presente estudo.

Observa-se em estudos sobre a percepção de qualidade de vida de estudantes do último ano de Medicina forte tendência de estes apresentarem baixos escores no domínio psicológi$\mathrm{Co}^{14,16,17}$. Este déficit de pontuação identificado nos estudantes de Medicina pode ser explicado pelo fato de que estar no último ano, culminando em um período estressante e delicado, no qual o estudante possui no seu dia-a-dia plantões médicos, preocupações com os exames da residência e decisões importantes sobre a carreira ${ }^{18,19}$. A ansiedade, depressão e problemas com o sono se apresentam como sintomas de déficits no domínio psicológico e constituem-se em fatores de risco para o acometimento de patologias mais graves, podendo prejudicar a qualidade profissional do futuro médico.

Tabela 2: Frequências e percentagens das questões gerais (Q1 e Q2) do WHOQOL-bref dos estudantes de Medicina.

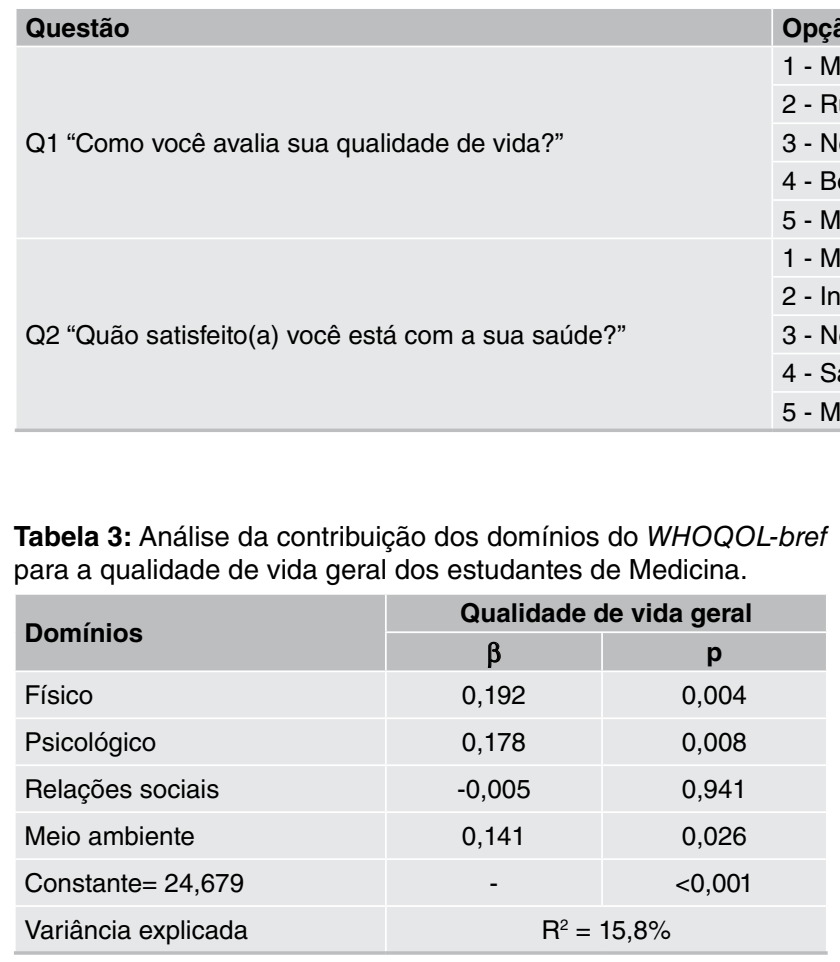

\begin{tabular}{|l|c|c|}
\hline Opção de resposta & $\mathbf{n}$ & $\mathbf{( \% )}$ \\
\hline 1 - Muito ruim & 0 & 0 \\
\hline 2 - Ruim & 14 & 4,7 \\
\hline 3 - Nem ruim nem boa & 75 & 24,9 \\
\hline 4 - Boa & 128 & 42,5 \\
\hline 5 - Muito boa & 84 & 27,9 \\
\hline 1 - Muito insatisfeito & 5 & 1,7 \\
\hline 2 - Insatisfeito & 13 & 4,3 \\
\hline 3 - Nem satisfeito nem insatisfeito & 66 & 21,9 \\
\hline 4 - Satisfeito & 99 & 32,9 \\
\hline 5 - Muito satisfeito & 118 & 39,2 \\
\hline
\end{tabular}

Tabela 3: Análise da contribuição dos domínios do WHOQOL-bref para a qualidade de vida geral dos estudantes de Medicina.
Tabela 4: Qualidade de vida considerando os estudantes com e sem dificuldade em conciliar internato/estudo.

\begin{tabular}{l|c|c|c|}
\hline Domínios & \multicolumn{2}{|c|}{$\begin{array}{c}\text { Dificuldade em conciliar } \\
\text { internato/estudo* }\end{array}$} & p \\
\hline Físico & Sim & Não & \\
\hline Psicológico & $75,0(17,9)$ & $78,6(16,7)$ & 0,026 \\
\hline Social & $75,0(17,5)$ & $75,0(11,5)$ & 0,077 \\
\hline Ambiental & $83,3(25,0)$ & $83,3(16,7)$ & 0,580 \\
\hline $\begin{array}{l}\text { Qualidade de } \\
\text { vida geral }\end{array}$ & $75,0(15,6)$ & $78,1(15,6)$ & 0,214 \\
\hline *Resultados expressos em mediana e intervalo interquartil. & \\
\hline
\end{tabular}


Ainda, foi identificado que, aproximadamente um terço da presente amostra, possuía avaliação neutra ou negativa da qualidade de vida. De acordo com estudo de Olmo et al. ${ }^{20}$, em que investigaram as diferenças na qualidade de vida de estudantes de Medicina do primeiro e último ano, observaram que os estudantes do primeiro ano apresentaram melhores condições psicológicas $(\mathrm{p}=0,034)$ e sociais ( $\mathrm{p}=0,011)$ quando comparados aos do último ano. Em estudo de mesmo objetivo, Alves et al. ${ }^{16}$, identificaram que os escores do domínio psicológico foram menores nos alunos do último período quando comparados aos do primeiro período $(\mathrm{p}=0,001)$. Pode-se supor que a percepção da qualidade de vida dos estudantes de Medicina, principalmente no que se refere ao domínio psicológico, tornasse mais negativa ao longo da graduação.

Estudantes com dificuldade de conciliar o internato com o estudo tiveram percepção mais negativa de qualidade de vida, especialmente no domínio físico. Estudo de Jamali et al ${ }^{21}$, com 1086 estudantes de Medicina iranianos, indicou que estar no internato mostrou associação significativa com menores escores do componente físico $(\mathrm{OR}=0,12 ; \mathrm{p}<0,001)$ e mental $(\mathrm{OR}=0,12 ; \mathrm{p}<0,001)$ quando comparados aos estudantes das fases iniciais. Menores escores de qualidade de vida em estudantes de internato podem ser atribuídos à maior prevalência de depressão em comparação aos estudantes nos níveis básicos e intermediários da educação médi$\mathrm{ca}^{22}$. Além disso, fatores como a elevada carga horária de trabalho nos hospitais, a responsabilidade pelos pacientes, erros médicos percebidos durante o treinamento, privação de sono, fadiga e transtornos do humor também podem contribuir para a redução da qualidade de vida nestes estudantes ${ }^{23}$.

Dentre as questões que apresentaram menores pontuações médias, a questão relacionada à satisfação com o sono foi a que apresentou segunda menor média. Altas prevalências de privação do sono são observadas em estudantes de Medicina, especialmente nos estudantes em período de internato ${ }^{24,25}$. Diante destes resultados destaca-se que, dentro do contexto dos problemas psíquicos, déficits de duração ou de qualidade do sono devem ser prevenidas.

Já em relação às contribuições dos domínios sobre a qualidade de vida, o domínio relações sociais foi o único que não apresentou valor significativo para explicar a qualidade de vida geral da presente amostra. Por outro lado, fatores do domínio físico, como dor física, fadiga, sono, dependência de tratamento (farmacológico e fisioterapêutico) e, capacidade de trabalho ${ }^{26}$, podem acarretar em diminuição na qualidade de vida e aumentar o nível de depressão ${ }^{27}$ destes estudantes. Assim como, fatores do domínio psicológico podem refletir sobre a vitalidade, saúde física e psíquica ${ }^{18}$, e o domínio ambiental evidenciar que o ambiente de trabalho pode influenciar no estilo de vida e na escolha por hábitos saudáveis ${ }^{28}$. Evidencia-se, desta forma, que os fatores dos respectivos domínios ajudam a explicar não só a qualidade de vida, mas, também, a influência das percepções positivas e negativas sobre a saúde dos sujeitos investigados.
Os pontos positivos destacados neste estudo foram a utilização de amostra representativa de estudantes de Medicina em período de internato de Santa Catarina - SC, a utilização de um instrumento validado internacionalmente (WHOQOL-bref) e promoção da discussão dos domínios que tem maior contribuição para a qualidade de vida e saúde destes estudantes. Apontam-se como limitações, variáveis que não foram contempladas neste estudo, como por exemplo, e eficiência do sono, depressão, nível de atividade física e secreção de cortisol. Além disso, há que se sugerir estudos que possam relacionar a qualidade de vida com a condição socioeconômica desses estudantes, já que o grau de dificuldade em aliar os estudos com o trabalho/ internato pode estar relacionado com os recursos econômicos que as famílias dispõem para adquirir material de estudo, transporte, espaço físico adequado para estudo, lazer; sendo, portanto, diretamente relacionados com a maneira como se aproveita o tempo disponível.

Deste modo, conclui-se que os piores indicadores de qualidade de vida foram os domínios Psicológico, Físico e a qualidade de vida geral. Uma parcela da amostra relatou percepção neutra ou negativa de sua qualidade de vida. Alunos com dificuldade em conciliar o internato e os estudos apresentam percepção mais negativa no domínio Física e na qualidade de vida geral.

Fatores físicos e que dizem respeito à qualidade de vida geral, acrescido a dificuldades em conciliar o trabalho exercido no internato e os estudos das disciplinas do curso de Medicina parecem ser os principais fatores para uma percepção mais negativa de qualidade de vida dos estudantes em internato do curso de Medicina. Estudos sobre a qualidade de vida são relevantes para os futuros profissionais da Medicina considerando que fatores relacionados à baixa qualidade de vida também costumam estar associados com o adoecimento. Além disso, considerando que os profissionais da saúde são interlocutores da promoção da saúde, nada mais coerente que incentivar para que os próprios profissionais se preocupem com a sua própria saúde, o que deve ser feito desde o momento em que são estudantes.

O estudo aponta para quase um terço com percepção regular ou negativa de qualidade de vida de estudantes no final do curso. Ainda, estudantes com dificuldade em conciliar os estudos e o internato apresentaram percepção mais negativa quanto ao domínio físico e na qualidade de vida geral. Assim, não se deve negligenciar a saúde desses futuros profissionais que podem, inclusive, estar se questionando quanto à manutenção e promoção da saúde, uma vez que não se nota por parte das instituições de ensino nenhuma ação voltada para a avaliação ou promoção da qualidade de vida desses estudantes. Sugerese um acesso com horário e local definidos para atendimento exclusivo aos estudantes, de maneira que fosse garantido o sigilo e que os mesmos pudessem ser acolhidos e atendidos de maneira integral. 


\section{REFERÊNCIAS}

1. World Health Organization (WHO). The WHOQOL group. Measuring quality of life. Disponível em: http://www.who.int/mental health/media/68.pdf. Acesso em: 22 mar 2018.

2. Pereira EF, Teixeira CS, Santos A. Qualidade de vida: abordagens, conceitos e avaliação. Rev Bras Edu Fís Esporte. 2012;26(2):24150.

http://dx.doi.org/10.1590/S1807-55092012000200007

3. Figueiredo AM, Ribeiro GM, Reggiani ALM, Pinheiro BA, Leopoldo GO, Duarte JAH, et al. Percepções dos estudantes de medicina da UFOP sobre sua qualidade de vida. Rev Bras Educ Med. 2014;38(4):435-43.

http://dx.doi.org/10.1590/S0100-55022014000400004

4. Klemenc-Ketis Z, Kersnik J, Eder K, Colaric D. Factors associated with health-related quality of life among university students. Srp Arh Celok Lek. 2011;139(3-4):197-202.

5. Pekmezovic T, Popovic A, Tepavcevic DK, Gazibara T, Paunic M. Factors associated with health-related quality of life among Belgrade University students. Qual Life Res. 2011;20:391-7. http://dx.doi.org/10.1007/s11136-010-9754-x

6. Maynard PL, Rohrer JE, Fulton L. Health-related quality of life among online university students. J Prim Care Community Health. 2015;6(1):48-53.

http://dx.doi.org/10.1177/2150131914545517

7. Scholz M, Neumann C, Steinmann C, Hammer CM, Schröder A, Ebel N, et al. Development and correlation of work-related behavior and experience patterns, burnout and quality of life in medical students from their freshmanship to the first state examination. Psychother Psychosom Med Psychol. 2015; 65(3-4):93-8.

http://dx.doi.org/10.1055/s-0034-1375630

8. Chazan ACS, Campos MR, Portugal FB. Qualidade de vida de estudantes de medicina da UERJ por meio do WHOQOLbref: uma abordagem multivariada. Ciênc Saúde Coletiva. 2015;20(2):547-56.

http://dx.doi.org/10.1590/1413-81232015202.05182014

9. Zhang Y, Qu B, Lun S, Wang D, Guo Y, Liu J. Quality of life of medical students in China: a study using the WHOQOL-BREF. PloS One. 2012;7(11):e49714.

https://dx.doi.org/10.1371/journal.pone.0049714

10. Diderichsen S, Andersson J, Johansson EE, Verdonk P, LagroJanssen A, Hamberg K. Swedish medical students' expectations of their future life. Int J Med Educ. 2011;2:140-6.

https://dx.doi.org/10.5116/ijme.4ec5.92b8

11. Barbetta PA. Estatística aplicada às ciências sociais: UFSC; 2008.

12. The WHOQOL Group. The development of the World Health Organization quality of life assessment instrument (the WHOQOL). In: Orley J, Kuyken W. Quality of Life Assessment: International Perspectives. Springer: 1994;41-57.

https://dx.doi.org/10.1007/978-3-642-79123-9 4

13. Fleck MPA, Louzada S, Xavier M, Chachamovich E, Vieira G, Santos $L$, et al. Aplicação da versão em português do instrumento abreviado de avaliação da qualidade de vida" WHOQOL-bref". Rev Saúde Pública. 2000;34(2):178-83. http://dx.doi.org/10.1590/S0034-89102000000200012
14. Nayak MSDP, Naidu SA, Krishnaveni A, Sreegiri S, Srinivas PJ. Quality Of Life in Medical Students of Andhra Medical College, Visakhapatnam. Int J Health Sci Res. 2014;4(12):39-43.

15. Bampil LNS, Baraldil S, Guilheml D, Araújo MP, Campos ACO. Qualidade de vida de estudantes de Medicina da Universidade de Brasília. Rev Bras Educ Med. 2013;37(2):217-25.

16. Alves JGB, Tenório M, Anjos AG, Figueroa JN. Qualidade de vida em estudantes de Medicina no início e final do curso: avaliação pelo WHOQOL-bref. Rev Bras Educ Med. 2010;34(1):91-6. http://dx.doi.org/10.1590/S0100-55022010000100011

17. Paro CA, Bittencourt ZZLC. Quality of life of the undergraduate health students. Rev Bras Educ Med. 2013;37(3):365-75. http://dx.doi.org/10.1590/S0100-55022013000300009

18. Raj SR, Simpson CS, Hopman WM, Singer MA. Health-related quality of life among final-year medical students. CMAJ. 2000;162(4):509-10.

19. Willcock SM, Daly MG, Tennant CC, Allard BJ. Burnout and psychiatric morbidity in new medical graduates. Med $\mathrm{J}$ Aust. 2004;181(7):357-60.

20. Olmo NRS, Ferreira LF, Prado AD, Martins LC, Dedivitis RA. Percepção dos estudantes de medicina do primeiro e sexto anos quanto à qualidade de vida. Diagn tratamento. 2012;17(4):157-61 .

21. Jamali A, Tofangchiha S, Jamali R, Nedjat S, Jan D, Narimani A, et al. Medical students' health-related quality of life: roles of social and behavioural factors. Med Educ. 2013;47(10):1001-12. http://dx.doi.org/10.1111/medu.12247

22. Baldassin S, Alves TC, Andrade AG, Martins LAN. The characteristics of depressive symptoms in medical students during medical education and training: a cross-sectional study. BMC Med Educ. 2008;8:60.

http://dx.doi.org/10.1186/1472-6920-8-60

23. Bellini LM, Baime M, Shea JA. Variation of mood and empathy during internship. Jama. 2002;287(23):3143-6. http://dx.doi.org/10.1001/jama.287.23.3143

24. Nojomi M, Bandi MFG, Kaffashi S. Sleep pattern in medical students and residents. Arch Iran Med. 2009;12(6):542-9.

25. Waqas A, Khan S, Sharif W, Khalid U, Ali A. Association of academic stress with sleeping difficulties in medical students of a Pakistani medical school: a cross sectional survey. Peer J. 2015;3:e840.

http://dx.doi.org/10.7717/peerj.840

26. Mendonça AEO, Salvetti MG, Maia EMC, Silva ACO, Torres GV. Análise dos aspectos físicos da qualidade de vida de receptores de rim. Rev Esc Enferm USP. 2015;49(1):76-81. http://dx.doi.org/10.1590/S0080-623420150000100010

27. Goldin SB, Wahi MM, Farooq OS, Borgman HA, Carpenter HL, Wiegand LR, et al. Student quality-of-life declines during third year surgical clerkship. J Surg Res. 2007;143(1):151-7. http://dx.doi.org/10.1016/j.jss.2007.08.021

28. Florindo AA, Salvador EP, Reis RS, Guimarães VV. Perception of the environment and practice of physical activity by adults in a low socioeconomic area. Rev Saude Publica. 2011;45(2):302-10. http://dx.doi.org/10.1590/S0034-89102011000200009 